\title{
UNIVERSAL LUSIN MEASURABILITY AND SUBFAMILY SUMMABLE FAMILIES IN ABELIAN TOPOLOGICAL GROUPS
}

\author{
WILLIAM H. GRAVES
}

\begin{abstract}
It is proved that if $G$ is a Hausdorff abelian topological group with respect to topologies $\alpha \subseteq \beta$ such that $\beta$ is complete and the identity map of $(G, \alpha)$ onto $(G, \beta)$ is universally Lusin measurable, then the subfamily summable families are the same for $\alpha$ and $\beta$.
\end{abstract}

0. Introduction. Kalton's remarkable paper [5] was the forerunner among other recent entrants in a newly directed study of the Orlicz-Pettis theorem. The papers [5], [6] of Kalton, [3], [4] of Drewnowski, [2] of Christensen, [1] of Andersen and Christensen, and [7], [8], [9] of Labuda are part of a shift from weak-implies-how-strong studies to strong-is-implied-by-how-weak studies. In the last of these papers is established a result conjectured by Thomas in [12]. The purpose of this paper is to give a very simple proof, based on nothing deeper than Fubini's theorem, of a variation on the conjecture of Thomas. It is here proved that if $G$ is a Hausdorf abelian topological group with respect to two topologies $\alpha, \beta$ such that $\alpha \subseteq \beta, \beta$ is complete, and the identity map $9:(G, \alpha) \rightarrow(G, \beta)$ is universally Lusin measurable, then the subfamily summable families in $G$ are the same for $\alpha$ and $\beta$. Labuda's version of this in [9] is set in the category of Hausdorff topological vector spaces, assumes universal measurability of $g$ rather than universal Lusin measurability, draws upon deeper results than does the version proved herein, and may be obtained in generalized form from the main result here.

The assistance of both the referee and Iwo Labuda in correcting and strengthening the results of $\S 3$, initially in muddled form, is gratefully acknowledged. Also acknowledged is receipt of a preprint, after acceptance of this paper, is which the main result here had also been earlier established by Jan Pachl using a technique very similar to that used here. His precedence in this matter is acknowledged.

1. Preliminaries. Let $S$ be an infinite set. $\mathcal{P}(S)=\{0,1\}^{S}$ is the topological product of $S$ copies of the discrete space $\{0,1\}$ and so is a compact Hausdorff space. The power set of $S$ will be identified throughout with $\mathscr{P}(S)$, and no distinction will be made between a set and its characteristic function.

Received by the editors December 22, 1977.

AMS (MOS) subject classifications (1970). Primary 28A45, 46G10.

Key words and phrases. Orlicz-Pettis theorem, subfamily summable families, abelian group-valued measures, universal measurability. 
It is with this understanding that set-theoretic terms such as $a \cup b$ and $a \backslash b$ are defined for $a, b \in \mathcal{P}(S)$. For $s \in S, e_{s}$ is the characteristic function of $\{s\}$.

$G \in \mathcal{G} \mathscr{R} \mathcal{P}$ means that $G$ is a Hausdorff abelian topological group. $\Phi$ : $\mathscr{P}(S) \rightarrow G$ is additive if $\Phi(a \cup b)=\Phi(a)+\Phi(b)$ for $a, b \in \mathscr{P}(S)$ such that $a \cap b=0$. For additive $\Phi$ and $s \in S$, let $\Phi_{s}=\Phi\left(e_{s}\right)$. If additive $\Phi$ is continuous, then $\left\{\Phi_{s}\right\}=\left\{\Phi_{s}: s \in S\right\}$ is a subfamily summable family in $G$ in that $\left\{\Phi_{s}: s \in S^{\prime}\right\}$ is summable for every $S^{\prime} \subseteq S\left(\Phi(a)=\sum_{s \in a} \Phi_{s}\right.$ for $a \in$ $\mathcal{P}(S)$ ). Conversely, if $\left\{\Phi_{s}\right\}$ is a subfamily summable family in $G$, then $\Phi(a)=\sum_{s \in a} \Phi_{s}$ defines an additive, continuous map $\Phi: \mathcal{P}(S) \rightarrow G$. With these conventions of identification, this equivalence can be condensed.

1.1. Proposition. Let $\Phi: \mathscr{P}(S) \rightarrow G \in \mathcal{G} \Re \mathcal{P}$ be additive. Then $\Phi$ is continuous if and only if $\left\{\Phi_{s}\right\}$ is subfamily summable and $\Phi(a)=\sum_{s \in a} \Phi_{s}$ for each $a \in \mathscr{P}(S)$.

It is well known that in the special case $S=\mathbf{N}$, the set of positive integers, a sequence $\left(\Phi_{n}\right)$ is subfamily summable if and only if it is subseries convergent in that $\Sigma \Phi_{n_{i}}$ converges for every subsequence $\left(\Phi_{n_{i}}\right)$ of $\left(\Phi_{n}\right)$. So 1.1 has special form in this case.

1.2. Proposition. Let $\Phi: \mathscr{P}(\mathbf{N}) \rightarrow G \in \mathcal{G} \Re \mathcal{P}$ be additive. Then $\Phi$ is continuous if and only if $\left(\Phi_{n}\right)$ is subseries convergent and $\Phi(a)=\sum_{n \in a} \Phi_{n}$ for each $a \in \mathscr{P}(\mathbf{N})$.

Additive $\Phi: \mathscr{P}(S) \rightarrow G \in \mathcal{G} \mathscr{R} \mathscr{P}$ is exhaustive if $\Phi\left(a_{n}\right) \rightarrow 0$ for every sequence $\left(a_{n}\right)$ of pairwise disjoint members of $\mathscr{P}(S)$.

All topological spaces will be Hausdorff. For such $X$, let $\mathfrak{B}_{X}$ be the $\sigma$-algebra of all Borel subsets of $X$ (generated by the open subsets). $\mu \in$ $\mathcal{R}(X)$ means that $\mu$ is a finite nonnegative Radon measure on $X$; that is, $\mu$ : $\mathscr{B}_{X} \rightarrow \mathbf{R}^{+}$is countably additive and both open regular and compact regular. So when $X$ is compact, $\Re(X)$ consists of all regular Borel measures in the usual sense. For $\mu \in \mathcal{R}(X), M \subseteq X$ is $\mu$-measurable if $B_{1} \subseteq M \subseteq B_{2}$ for some $B_{1}, B_{2} \in \mathscr{B}_{X}$ such that $\mu\left(B_{2}\right)-\mu\left(B_{1}\right)=0$, and $M_{\mu}$ is the $\sigma$-algebra of all such $M$. For $\mu \in \mathfrak{R}(X), f: X \rightarrow Y$ is $\mu$-measurable if $f^{-1}\left(\mathscr{B}_{Y}\right) \subseteq M_{\mu}$ and is Lusin $\mu$-measurable if for $\varepsilon>0$ and compact $K \subseteq X$ there is compact $K_{\varepsilon} \subseteq K$ such that $\mu(K)-\mu\left(K_{\varepsilon}\right)<\varepsilon$ and $f$ is continuous on $K_{\varepsilon} . f$ is universally measurable if $\mu$-measurable for all $\mu \in \mathcal{R}(X)$ and is universally Lusin measurable if Lusin $\mu$-measurable for all $\mu \in \Re(X)$.

The next two results are but special cases of more general results available in [11]. Proofs are included here in order that the simplicity of the results in the next section will be evident.

1.3. LeMmA. For compact $K \subseteq X$, continuous $f: K \rightarrow Y$, and $\mu \in \mathcal{R}(X)$, define $f(\mu): \mathscr{B}_{Y} \rightarrow \mathbf{R}$ by $f(\mu)(B)=\mu\left(f^{-1}(B)\right), B \in \mathscr{B}_{Y}$. Then $f(\mu) \in \mathscr{R}(Y)$.

Proof. $f(\mu)$ is well defined since $f^{-1}\left(\mathscr{B}_{Y}\right) \subseteq \mathscr{B}_{K} \subseteq \mathscr{B}_{X}$ and is clearly 
countably additive. Consider $B \in \mathscr{B}_{Y}$ and $\varepsilon>0$. Pick compact $K_{0} \subseteq f^{-1}(B)$ such that $\mu\left(f^{-1}(B)\right)-\mu\left(K_{0}\right)<\varepsilon$. Then $f\left(K_{0}\right)$ is compact, $f\left(K_{0}\right) \subseteq B$, and $f(\mu)(B)-f(\mu)\left(f\left(K_{0}\right)\right)<\varepsilon$, proving that $f(\mu)$ is compact regular. Choose open $V \supseteq f^{-1}(B)$ such that $\mu(V)-\mu\left(f^{-1}(B)\right)<\varepsilon$. Then $f(K \backslash V \cap K)$ is compact, and $V_{1}=Y \backslash f(K \backslash V \cap K)$ is open as is $f^{-1}\left(V_{1}\right)$. But $f^{-1}(B) \subseteq$ $f^{-1}\left(V_{1}\right) \subseteq V \cap K$ and $f(\mu)\left(V_{1}\right)-f(\mu)(B)<\varepsilon$, proving that $f(\mu)$ is open regular.

1.4. Proposition. If $f: X \rightarrow Y$ is continuous and $g: Y \rightarrow Z$ is universally Lusin measurable, then $g \circ f$ is universally Lusin measurable.

Proof. Consider $\mu \in \mathcal{R}(X)$, compact $K$, and $\varepsilon>0$. Let $f_{K}$ be the restriction of $f$ to $K$. Then $f_{K}(\mu) \in \Re(Y)$ by 1.3. Choose compact $C_{\varepsilon} \subseteq f(K)$ such that $f_{K}(\mu)(f(K))-f_{K}(\mu)\left(C_{\varepsilon}\right)<\varepsilon$ and $g$ is continuous on $C_{\varepsilon}$. Let $K_{\varepsilon}=$ $f_{K}^{-1}\left(C_{\varepsilon}\right)$. Then $K_{\varepsilon}$ is compact, $g \circ f$ is continuous on $K_{\varepsilon}$, and $\mu(K)-\mu\left(K_{\varepsilon}\right)<$ $\varepsilon$.

2. Key lemma and main results. Let $\mu$ be the Radon measure defined on the discrete space $\{0,1\}$ by $\mu(\{0\})=\frac{1}{2}=\mu(\{1\})$. Let $\lambda$ be the associated product measure on $\{0,1\}^{\mathbf{N}}=\mathscr{P}(\mathbf{N})$, also a Radon measure. Let $n \in \mathbf{N}$. Let $\mathscr{P}(\mathbf{N} \backslash n)$ $=\{0,1\}^{\mathbf{N} \backslash\{n\}}$, and let $\lambda_{n}$ be the product Radon measure on $\mathcal{P}(\mathbf{N} \backslash n)$ determined by $\mu$. Then $\mathscr{P}(\mathbf{N})=\{0,1\} \times \mathscr{P}(\mathbf{N} \backslash n)$, and for a Borel set $A \subseteq$ $\mathscr{P}(\mathbf{N})$, Fubini's theorem gives

$$
\lambda(A)=\int \mu\left(A_{c}\right) d \lambda_{n}(c)
$$

where for $c \in \mathscr{P}(\mathbf{N} \backslash n)$,

$$
A_{c}=\{k \in\{0,1\}:(k, c) \in A\}
$$

is the $c$-section of $A$.

2.1. Lemma. If $\Phi: \mathcal{P}(\mathbf{N}) \rightarrow G \in \mathcal{G} \Re \mathcal{P}$ is additive and Lusin $\lambda$-measurable, then $\Phi_{n} \rightarrow 0$.

Proof. Choose compact $K \subseteq \mathscr{P}(\mathbf{N})$ such that $\lambda(\mathscr{P}(\mathbf{N}) \backslash K)<\frac{1}{2}$ and $\Phi$ is continuous on $K$. For $n \in \mathbf{N}$, let

$$
K_{n}=\left\{a \in K: a \cup e_{n}, a \backslash e_{n} \in K\right\},
$$

and let $A=K \backslash K_{n} . A$ is a Borel set since $K_{n}$ is compact. For $c \in \mathcal{P}(\mathbf{N} \backslash n)$, the $c$-section $A_{c}$ has at most one element, and so $\lambda(A) \leqslant \frac{1}{2}$ by Fubini's theorem. But

$$
\frac{1}{2}<\lambda(K)=\lambda(A)+\lambda\left(K_{n}\right)
$$

whence $\lambda\left(K_{n}\right)>0$ proving that $K_{n} \neq \varnothing$ for each $n \in \mathbf{N}$. Choose $a_{n} \in K_{n}$ for each $n \in \mathbf{N}$. If $\lim \Phi_{n} \neq 0$, there is a 0 -neighborhood $\Re$ in $G$ and a subsequence $\Phi_{n_{k}} \notin \Re$. Since $K$ is compact, there exist $a \in K$ and a subsequence $\left(a_{n_{k_{k}}}\right)$ of $\left(a_{n_{k}}\right)$ converging to $a$. But $a_{n} \cup e_{n}=\left(a_{n} \backslash e_{n}\right) \cup e_{n}$ is a 
disjoint union for all $n$, both

$$
a_{n_{k_{i}}} \cup e_{n_{k_{i}}} \rightarrow a \text { and } a_{n_{k_{i}}} \backslash e_{n_{k_{i}}} \rightarrow a
$$

as $i \rightarrow \infty$, and for each $i$,

$$
a_{n_{k_{i}}} \cup e_{n_{k_{i}}}, \quad a_{n_{k_{i}}} \backslash e_{n_{k_{i}}} \in K
$$

by definition of the $a_{n}$ 's. So by continuity of $\Phi$ on $K$,

$$
\Phi_{n_{k_{i}}}=\Phi\left(a_{n_{k_{i}}} \cup e_{n_{k_{i}}}\right)-\Phi\left(a_{n_{k_{i}}} \backslash e_{n_{k_{i}}}\right) \rightarrow 0
$$

contradicting $\Phi_{n_{k_{i}}} \notin \Re$.

2.2. ThEOREM. If $\Phi: \mathcal{P}(S) \rightarrow G \in \mathcal{G} \Re \mathcal{P}$ is additive and universally Lusin measurable, then $\Phi$ is exhaustive.

Proof. If not, then $\Phi\left(a_{n}\right) \notin \Re$ for some sequence $\left(a_{n}\right)$ of pairwise disjoint subsets of $S$ and some 0 -neighborhood $\Re$ in $G$. Define a continuous map $f$ : $\mathscr{P}(\mathbf{N}) \rightarrow \mathscr{P}(S)$ by $f(b)=\cup_{n \in b} a_{n}$ and let $\Psi=\Phi \circ f$. Then $\Psi$ is universally Lusin measurable by 1.4 . So $\Phi\left(a_{n}\right)=\Psi_{n} \rightarrow 0$ by 2.1 , a contradiction.

2.3. Corollary. For $(G, \alpha),(G, \beta) \in \mathcal{G} \Re \mathcal{P}$, assume that $\alpha \subseteq \beta$, that $(G, \beta)$ is complete, and that the identity map $G:(G, \alpha) \rightarrow(G, \beta)$ is universally Lusin measurable. Then the subfamily summable families are the same for $(G, \alpha)$ and $(G, \beta)$.

Proof. It suffices to prove that an $\alpha$-subfamily summable family $\left\{\Phi_{s}\right\}$ is $\beta$-subfamily summable. Let $\Phi: \mathcal{P}(S) \rightarrow(G, \alpha)$ be the associated continuous, additive function. Let $\Phi_{\beta}=9 \circ \Phi$. Then $\Phi_{\beta}$ is universally Lusin measurable by 1.4 and so is $\beta$-exhaustive by 2.2 . Since $\beta$ is complete, $\alpha \subseteq \beta$, and $\left\{\Phi_{s}\right\}$ is $\alpha$-subfamily summable, it follows that it is $\beta$-subfamily summable.

It is clear from the above proof that the special case $S=\mathbf{N}$ of 2.3 requires only $\beta$-sequential completeness.

2.4. Corollary. For $(G, \alpha),(G, \beta) \in \mathcal{G} \Re \mathscr{P}$, assume that $\alpha \subseteq \beta$, that $(G, \beta)$ is sequentially complete, and that the identity map $G:(G, \alpha) \rightarrow(G, \beta)$ is universally Lusin measurable. Then the subseries convergent series are the same for $(G, \alpha)$ and $(G, \beta)$.

The next corollary of 2.2 is a variant of a well-known theorem of Banach.

2.5. Corollary. Let $G \in \mathcal{G} \Re \mathcal{P}$ have topology determined by a complete, invariant metric. If a homomorphism $f: G \rightarrow G^{\prime} \in \mathcal{G} \Re \mathcal{P}$ is universally Lusin measurable, then it is continuous.

Proof (LABUda). If $f$ is not continuous, then $f\left(g_{n}\right) \notin \Re$ for some 0 -neighborhood $\Re$ in $G^{\prime}$ and some sequence $\left(g_{n}\right)$ in $G$ such that $g_{n} \rightarrow 0$. By completeness and invariance of the metric on $G$, there is a subsequence $\left(g_{n_{i}}\right)$ which is subseries convergent and hence determines a continuous, additive map $\Phi: \mathcal{P}(\mathbf{N}) \rightarrow G$ such that $\Phi_{i}=g_{n_{i}}$. Then $\Psi=f \circ \Phi$ is additive and exhaustive by 1.4 and 2.2. Thus, $f\left(g_{n_{i}}\right)=\Psi_{i} \rightarrow 0$, a contradiction. 
3. Further results and connections with other work. Except for the reference to Fubini's theorem in the proof of 2.2, this paper has been self-contained and erected on a very elementary base up to this point. When restricted to the category of topological vector spaces. 2.2-2.5 follow from Labuda's deeper work in [9] since universal measurability follows from universal Lusin measurability. In order to generate Kalton's result in [5] and results of Labuda in [9] from 2.2-2.5, it is necessary to invoke measurability considerations deeper than those in $\S 1$. These measurability results are listed below.

Recall that $X$ is $K$-analytic if it is the continuous image of a $K_{\sigma \delta}$ subspace of some space and that $X$ is Suslin if it is the continuous image of a separable, complete metric space. A locally convex topological vector space is Fréchet if its topology is that of a complete, invariant metric.

3.1. Theorem. For $\mu \in \mathcal{R}(X)$, if $f: X \rightarrow Y$ is $\mu$-Lusin measurable, then it is $\mu$-measurable. The converse is true if $Y$ is Suslin.

Proof. See [11, Theorem 5, p. 26 and Theorem 14, p. 129].

3.2. TheOREM. If topology $\alpha$ on $X$ is coarser than topology $\beta$ and $(X, \beta)$ is $K$-analytic, then the identity map $G:(X, \alpha) \rightarrow(X, \beta)$ is universally Lusin measurable.

Proof. See [11, Corollary 1, p. 126].

3.3. THEOREM. If $E$ is a Fréchet space, then the identity map $9:(E$, weak $) \rightarrow$ $E$ is universally Lusin measurable.

Proof. See [11, Theorem 3, p. 162].

3.4. TheOREM. Let $G \in \mathcal{G} \mathcal{P} \Re$ be complete and $K$-analytic. The subfamily summable families coincide for all Hausdorff topologies coarser than the original one and compatible with the group structure.

Proof. Apply 2.3 and 3.2. For Kalton's version, see [5, Theorem 3].

3.5. THEOREM. If $\Phi: \mathcal{P}(S) \rightarrow G \in \mathcal{G} \mathscr{R} \mathcal{P}$ is additive and universally measurable, then $\Phi$ is exhaustive.

Proof (LABUdA). $G$ may be embedded in a product, $\Pi G_{\gamma}$, of complete metrizable abelian groups. For each $\gamma$, let $\Phi_{\gamma}$ be the composition of $\Phi$ with the projection of $G$ on $G_{\gamma}$. Then $\Phi_{\gamma}$ is universally measurable and, by a result of Sazonov [10, Corollaries 1 and 2 of Theorem 11], is even $\mu$-essentially separably valued for every Radon measure $\mu$ on $\mathscr{P}(S)$. So by 3.1 , each $\Phi_{\gamma}$ is $\mu$-Lusin measurable for all such $\mu$, and the result now follows from 2.2.

Now Labuda's main result in [9], generalized to groups, follows from 3.5 just as 2.3 follows from 2.2 since $G \circ \Phi$ is universally measurable when $\Phi$ is continuous and 9 is universally measurable, [9, Proposition 1.3]. 
3.6. Corollary. For $(G, \alpha),(G, \beta) \in \mathcal{G} \Re \mathcal{P}$, assume that $\alpha \subseteq \beta$, that $(G, \beta)$ is complete, and that the identity map $G:(G, \alpha) \rightarrow(G, \beta)$ is universally measurable. Then the subfamily summable families are the same for $(G, \alpha)$ and $(G, \beta)$.

3.7. THEOREM. If $E$ is a Fréchet space, then the subfamily summable families (in particular, the subseries convergent series) are the same for the weak and the original (= strong) topology.

Proof. This follows from 2.3 and 3.3 and is the classical Orlicz-Pettis theorem.

A glance at its proof reveals that 2.5 can be strengthened via 3.6.

3.8. TheOREM. Let $G \in \mathcal{G} \Re \mathcal{P}$ have topology determined by a complete, invariant metric. If a homomorphism $f: G \rightarrow G^{\prime} \in \mathcal{G} \Re \mathcal{P}$ is universally measurable, then it is continuous.

\section{REFERENCES}

1. N. J. M. Andersen and J. P. R. Christensen, Some results on Borel structures with applications to subseries convergence in abelian topological groups, Israel J. Math. 15 (1973), 414-420.

2. J. P. R. Christensen, Borel structures and a topological zero-one law, Math. Scand. 29 (1971), 245-255.

3. L. Drewnowski, On the Orlicz Pettis type theorems of Kalton, Bull. Acad. Polon. Sci. Ser. Sci. Math. Astronom. Phys. 21 (1973), 515-518.

4. __ Another note on Kalton's theorems, Studia Math. 52 (1975), 233-237.

5. N. J. Kalton, Subseries convergence in topological groups and vector spaces, Israel J. Math. 10 (1971), 402-412.

6. Topologies on Riesz groups and applications to measure theory, Proc. London Math. Soc. (3) 28 (1974), 253-273.

7. I. Labuda, A generalization of Kalton's theorem, Bull. Acad. Polon. Sci. Sér. Sci. Math. Astronom. Phys. 21 (1973), 509-510.

8. Discrete copies of rings of sets in groups and Orlicz-Pettis theorem (preprint).

9. __ Universal measurability and summable families in tos (preprint).

10. V. V. Sazonov, On perfect measures, Amer. Math. Soc. Transl. (2) 48 (1965), 229-254.

11. L. Schwartz, Radon measures on arbitrary topological spaces and cylindrical measures, Oxford Univ. Press, New York and London, 1973.

12. E. Thomas, The Lebesgue-Nikodym theorem for vector-valued Radon measures, Mem. Amer. Math. Soc. No. 139 (1974).

Department of Mathematics, University of North Carolina, Chapel Hill, North Carolina 27514 